\title{
Apport de l'imagerie par résonance magnétique (IRM) dans le diagnostic du kyste hydatique cardiaque
}

\author{
A. ElMajhad, ${ }^{1}$ A. Lachhab, ${ }^{7}$ R.Cherradi, ${ }^{1}$ J.Srairi, ${ }^{7}$ N.Srairi, ${ }^{1}$ N. Doghmi ${ }^{1}$ et M. Cherti ${ }^{1}$
}

\section{Introduction}

Le kyste hydatique est une parasitose qui sévit à l'état endémique au Maroc. Une incidence de 4,55 cas opérés pour 100000 habitants a été évoquée dans le dernier rapport du Ministère de la Santé (2006), plaçant le Maroc en troisième position au Maghreb après la Tunisie et l'Algérie [1].

Bien qu'il n'existe pas de chiffres nationaux précis, l'atteinte cardiopéricardique reste une affection rare. Par contre, elle est caractérisée par la gravité de son évolution spontanée et la fréquence de ses complications qui sont le plus souvent révélatrices. Le diagnostic repose essentiellement sur les techniques d'imagerie (échocardiographie, tomodensitométrie, imagerie par résonance magnétique).

Nous rapportons dans ce travail les observations cliniques de deux jeunes patients atteints d'hydatidose cardio-péricardique en exposant la démarche diagnostique suivie. Nous espérons ainsi mettre la lumière sur les moyens diagnostiques d'imagerie dont disposent non seulement le clinicien pour confirmer l'affection, mais également le chirurgien pour mener à bien son geste curatif. Une attention particulière est donnée à l'imagerie par résonance magnétique (IRM), qui s'affirme comme moyen d'imagerie de première intention dans cette pathologie.

\section{Observation $n^{0} 1$}

Il s'agit d'un jeune patient de 16 ans, admis aux urgences du Centre hospitalier universitaire (CHU) Ibn Sina de Rabat (Maroc) en mars 2008 pour précordialgies à type de brûlures et crachats hémoptoïques remontant à 15 jours, avec une notion de contact avec les chiens à l'anamnèse.

L'examen clinique à l'admission retrouve un état hémodynamique stable ; un frémissement systolique au bord gauche du sternum est perçu, avec à l'auscultation cardiaque un souffle systolique rude et râpeux irradiant au bord gauche du sternum et dans le dos. L'électrocardiogramme objective un axe QRS en DIII, un hémibloc postérieur gauche et une hypertrophie ventriculaire droite. Le cliché thoracique de face montre un syndrome interstitiel basal bilatéral avec un cœur de volume normal. Biologiquement, on note une hyperéosinophilie.

Le patient a bénéficié d'une échographie abdominale qui a mis en évidence quatre kystes hydatiques hépatiques et un kyste hydatique rénal droit de siège. Les anomalies de l'examen cardio-vasculaire ont motivé la pratique d'une échocardiographie transthoracique objectivant une masse de $30 \mathrm{~mm}$ de diamètre au niveau de l'infundibulum pulmonaire responsable d'une obstruction de la voie d'éjection du ventricule droit (VD).
L'IRM permet de mettre en évidence une image kystique au niveau du septum infundibulaire avec un aspect bivésiculaire prolabant au niveau de la chambre de chasse du ventricule droit et mesurant $30 \times 20 \mathrm{~mm}$ (Figures 1-3).

Ainsi le diagnostic a été retenu sur les arguments anamnestique (contexte épidémiologique), biologique (hyperéosinophilie) et radiologique (kyste hydatique hépatique et rénal associé).

Sur le plan thérapeutique, le patient a été mis sous albendazole (400 mg par jour), puis a été confié aux chirurgiens pour geste curatif. L'ablation de la tumeur a étéfaite avec succès et les suites opératoires ont été simples. Le patient est toujours suivi en consultation.

\section{Observation $n^{\circ} 2$}

Le deuxième cas est celui d'une patiente de 36 ans, admise initialement au service de pneumologie du CHU Ibn Sina de Rabat (Maroc) en juin 2008. La patiente accusait des hémoptysies de moyenne abondance et une dyspnée. Une ponction pleurale a été décidée sur image kystique à la radiographie pulmonaire, compliquée d'une dissémination de l'hydatidose. Elle est réadmise aux urgences avec une dyspnée stade II- III de la New York Heart Association (NYHA). 

région infundibulaire

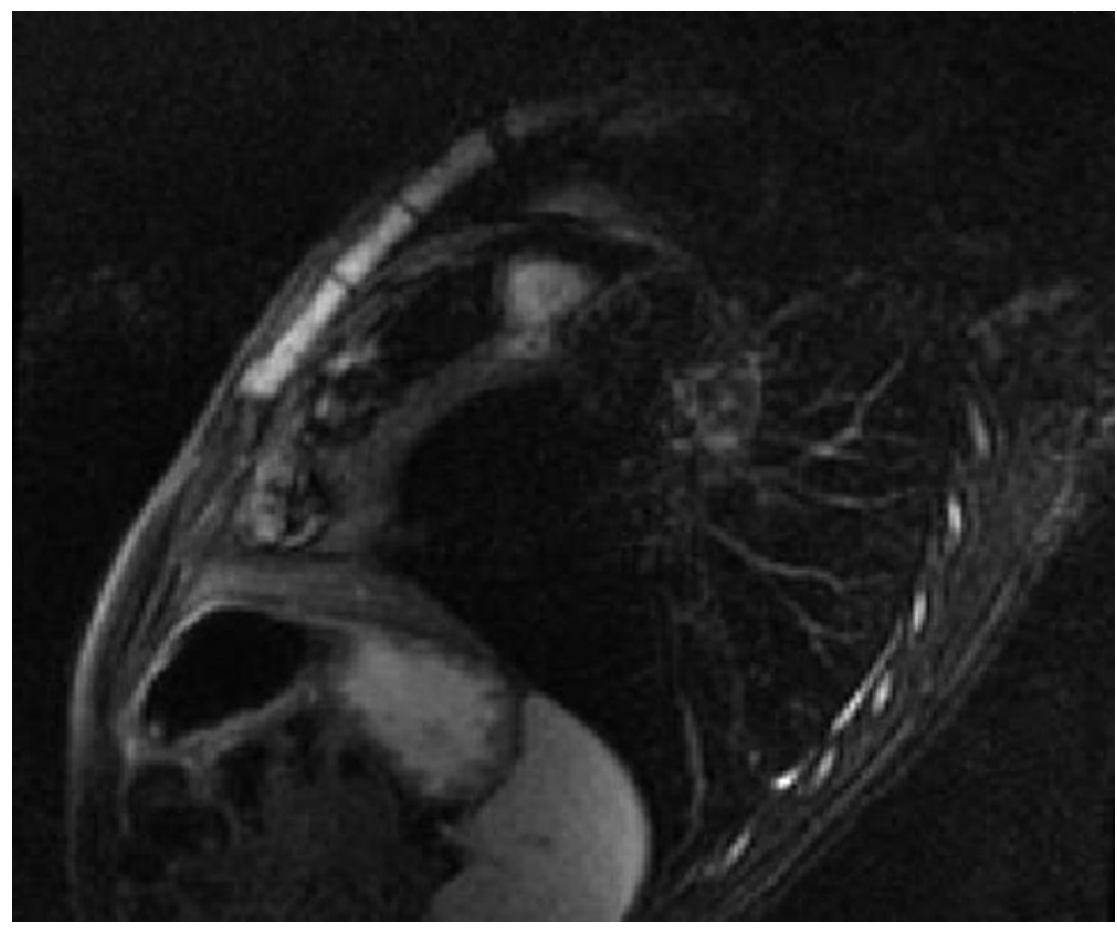

Figure 2 Coupe IRM passant par l'infundibulum pulmonaire montrant le kyste hydatique
L'examen clinique retrouve une légère cyanose, une tension artérielle à 95/60 mmHg. L'examen cardio-vasculaire relève un souffle systolique d'insuffisance tricuspidienne avec un éclat de B2 pulmonaire. L'électrocardiogramme s'inscrit en rythme régulier sinusal avec un axe QRS dévié à droite, un hémibloc postérieur gauche, une hypertrophie ventriculaire droite ainsi que des troubles diffus de la repolarisation secondaire. Le cliché thoracique laisse découvrir une image en lâcher de ballons avec une cardiomégalie V1 (Figure 4).

Bien que la dyspnée ait été rattachée aux lésions constatées à la radiographie pulmonaire, une exploration cardiaque a été proposée devant les anomalies de l'auscultation cardio-vasculaire et électrocardiographiques.

À l'échocardiographie, la pointe du VD est comblée par une masse kystique.

L'IRM cardiaque objective une formation kystique au niveau du VD mesurant $27 \times 36 \mathrm{~mm}$, adhérant à la paroi septale, à contenu impur sans aucun rehaussement (Figures 5-6).

Le kyste hydatique était adhérent au plan myocardique, contreindiquant tout geste chirurgical. La malade a été mise sous traitement médical exclusif (albendazole) prévu pour une durée de six mois. Elle a été malheureusement perdue de vue après quelques mois de suivi en consultation.

\section{Discussion}

L'hydatidose est une affection parasitaire assez fréquente qui constitue un problème de santé publique dans les zones d'endémie, notamment le pourtour du bassin méditerranéen.

La localisation cardio-péricardique est toutefois rare : 0,5 à $2 \%$ de l'ensemble des localisations, 2,6 \% des localisations hydatiques intra-thoraciques [2], le cour étant affecté par la circulation systémique 


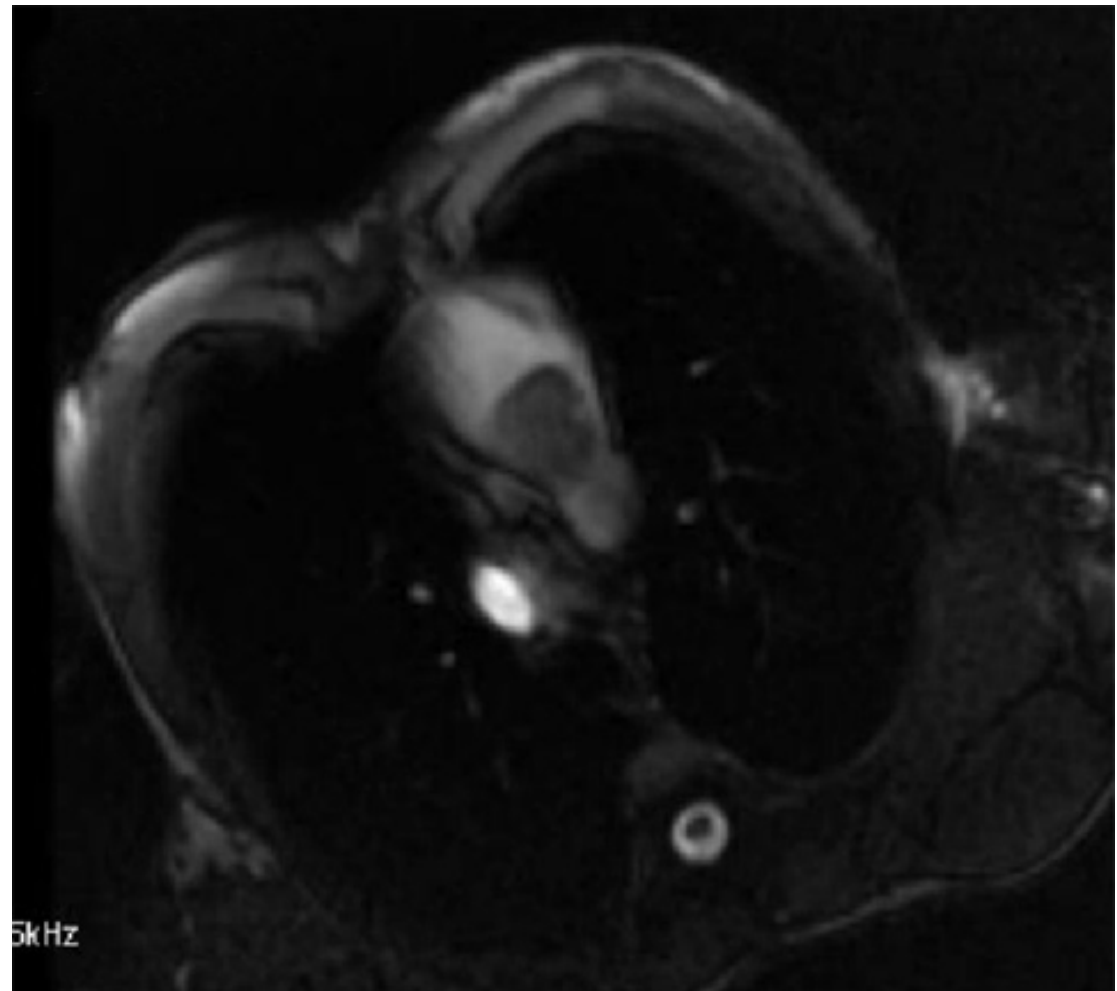

Figure 3 Coupe IRM infundibulaire en « sang noir » (black blood) montrant le kyste hydatique

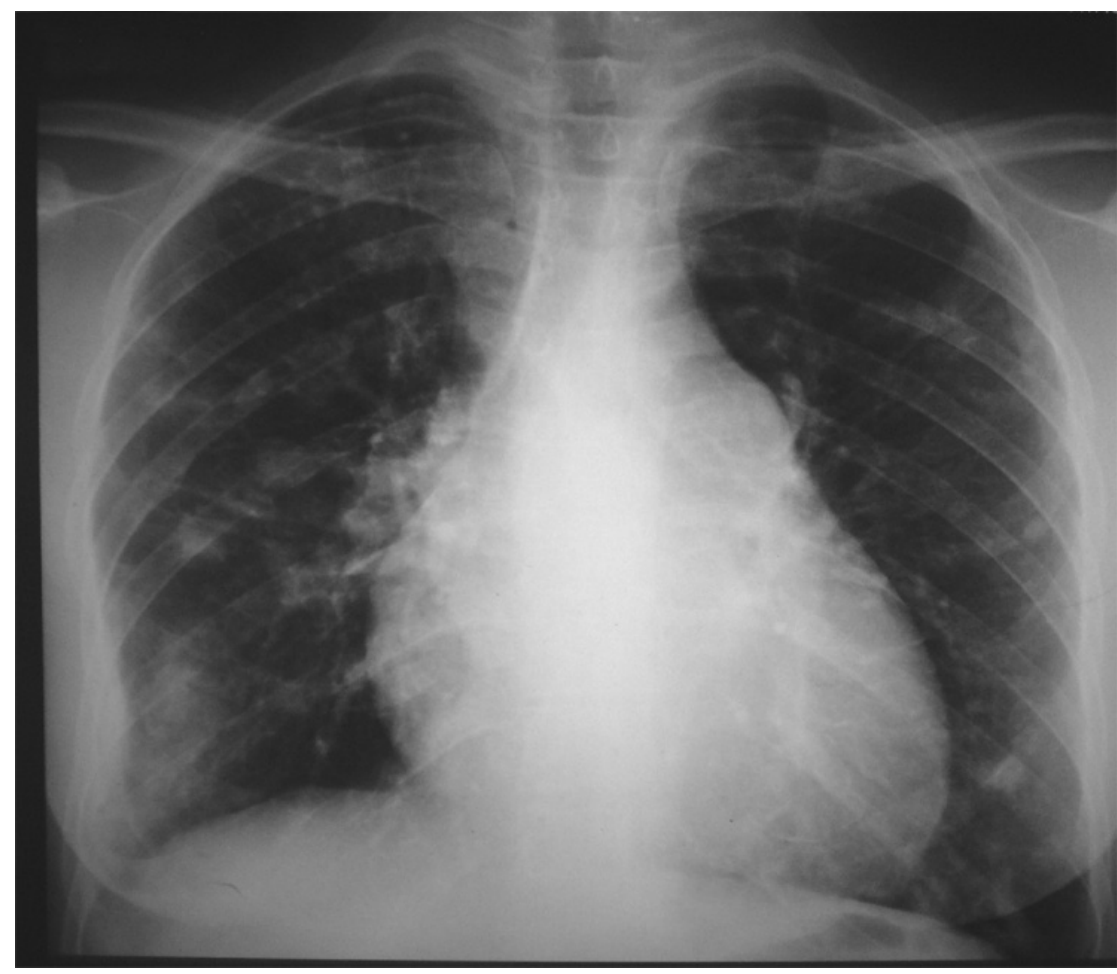

Figure 4 Radiographie pulmonaire de face montrant des images en lâcher de ballons ou pulmonaire, ou encore par extension à partir des organes de voisinage [3].

Le kyste hydatique se localise de préférence au niveau du ventricule gauche $(75 \%)$, suivi du ventricule droit $(15 \%)$, du septum interventriculaire $(10 \%)$, des oreillettes (5 à $8 \%$ ), et du péricarde dans (4\%) [4]. Dans nos deux cas, le kyste hydatique était localisé dans le ventricule droit.

Le kyste hydatique du cœur s'associe dans 20 à $40 \%$ des cas à d'autres localisations viscérales. C'est le cas chez nos deux patients (localisation pulmonaire chezl'un, hépatique et rénale chezl'autre).

Sur le plan clinique, la présentation de l'hydatidose cardiaque est souvent atypique et polymorphe, responsable d'un retard diagnostique [5]. La douleur thoracique est le symptôme le plus couramment rapporté [6]. Le mode de révélation le plus fréquent reste cependant la découverte fortuite par radiographie pulmonaire (qui peut montrer une voussure localisée, une déformation étendue d'un arc de la silhouette cardiaque, ou encore une hydatidose pulmonaire associée) ou par échographie transthoracique.

Chez notre premier patient, bien que la symptomatologie fût déroutante, le retard diagnostique est exclusivement dû au retard de consultation, les anomalies de l'examen clinique ayant rapidement motivé une exploration cardiaque menant au diagnostic.

Par contre, la présentation clinique atypique du deuxième cas a mené à une démarche diagnostique mal ciblée, conduisant à un retard diagnostique et une dissémination de la maladie.

L'échocardiographie Doppler transthoracique (ETT) ainsi que l'échocardiographie Doppler transœsophagienne (ETO) constituent les techniques d'imagerie de première intention dans le diagnostic des kystes 


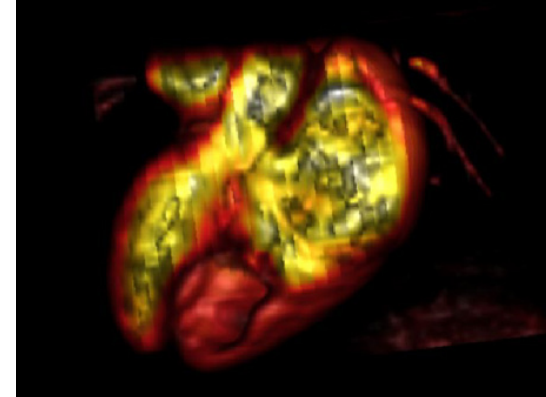

Figure 5 Image IRM de reconstruction montrant la pointe du VD comblée par le kyste hydatique

hydatiques cardiaques [6,7]. L'ETT reste un examen clé qui permet de visualiser le kyste, son siège, ses rapports et son retentissement. Néanmoins, les limites de cet examen sont représentées par l'échogénicité des patients et le diagnostic topographique précis.

La tomodensitométrie (TDM) ainsi que l'IRM complètent les données en fournissant des éléments plus pertinents concernant la topographie, la morphologie ainsi que le bilan d'extension [8].

L'IRM détermine les rapports exacts du kyste hydatique avec les structures cardiaques, la nature des constituants internes et externes du kyste, ainsi que l'extension de l'hydatidose en intra ou extra-thoracique [9].

L'IRM montre typiquement une lésion hypo-intense en T1, hyperintense en T2 avec hypo-signal périphérique en rapport avec la capsule fibreuse $[8,10]$.

Un nombre de tumeurs cardiaques doit être considéré comme diagnostics

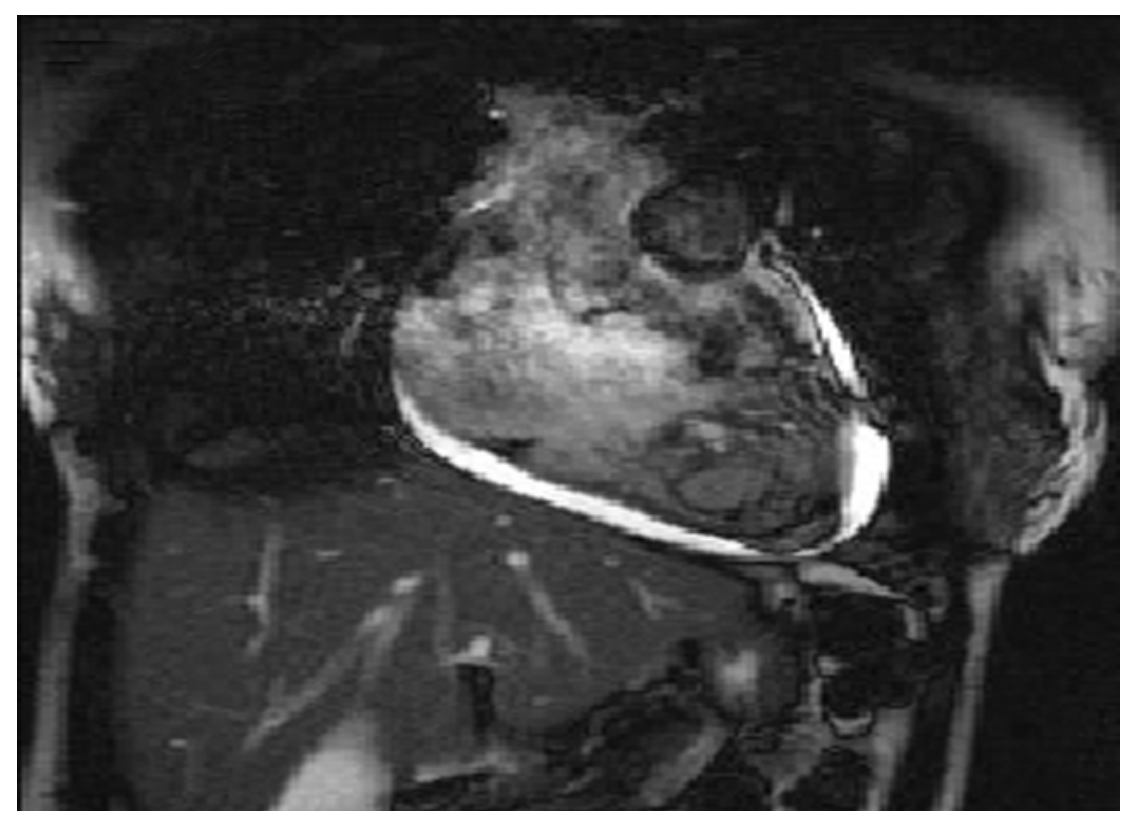

Figure 6 Coupe IRM 2 cavités le long du VD montrant le VD comblé depuis la région médio-ventriculaire jusqu'à l'apex par la masse hydatique avec une image kystique au niveau péricardique ainsi qu'un épanchement péricardique circonférentiel (IRM : imagerie par résonance magnétique ; VD : ventricule droit).

différentiels potentiels, notamment au stade pseudo-tumoral du kyste hydatique [11]. Cela dit, l'aspect multivésiculaire ou le détachement de membrane reste des éléments presque pathognomoniques $[12,13]$.

En somme, l'IRM permet le plus souvent non seulement d'assoir le diagnostic positif mais aussi de guider le geste chirurgical qui s'avère urgent $[5,14]$. Les antihelminthiques gardent une place de traitement adjuvant ou néo-adjuvant à la chirurgie, voire exclusif en cas de contre-indication formelle à celle-ci (le cas de notre deuxième patiente chez laquelle le kyste adhérait au plan myocardique).
L'IRM s'affirme également comme la technique d'imagerie de choix dans le suivi des malades, particulièrement dans la période post-opératoire.

\section{Conclusion}

Compte tenu de l'apport considérable de l'IRM dans le diagnostic et la décision thérapeutique des kystes hydatiques cardiaques, cet examen doit être sollicité devant toute suspicion de cette pathologie, menaçante par ses complications, et qui relève le plus souvent d'un geste chirurgical urgent.

\section{Références}

1. Ministère de la Santé, Direction de l'Épidémiologie et de Lutte contre les Maladies (DELM). Situation épidémiologique de I'hydatidose et activités réalisées en 2005 et 2006. Rapport annuel d'activités - État d'avancement des programmes de lutte contre les maladies parasitaires Années 2005 - 2006 (http:// www.sante.gov.ma/Departements/DELM/Maladies Parasitaires/Rapport Annuel des maladies parasitaires 2005-2006. pdf, consulté le 19 octobre 2011).

2. Perez-Gomez F et al. Cardiac echinococcosis: clinical pictures and complications. British Heart Journal, 1973, 35:1326-1331.
3. Ozer $\mathrm{N}$ et al. Hydatid cyst of the heart as a rare cause of embolit zation: report of 5 cases and review of published reports. Journal of the American Society of Echocardiography, 2001, 14:299-302.

4. Kaplan M et al. Cardiac hydatid cysts with intracavitary expansion. Annals of Thoracic Surgery, 2001, 71:1587-1590.

5. Onursal E et al. Surgical treatment of cardiac echinococcosis: report of eight cases. Surgery Today, 2001, 31:325-330.

6. Thameur $\mathrm{H}$ et al. Cardio-pericardial hydatid cysts. World Journal of Surgery, 2001, 25:58-67. 
7. Tufekcioglu $\mathrm{O}$ et al. Echocardiography findings in 16 cases of cardiac echinococcosis: proposal for a new classification system. Journal of the American Society of Echocardiography, 2007, 20:895-904

8. Kotoulas GK et al. Evaluation of hydatid disease of the heart with magnetic resonance imaging. Cardiovascular and Interventional Radiology, 1996, 19:187-189.

9. Oueslati $\mathrm{S}$ et al. Imagerie du kyste hydatique du cœur. À propos de 8 observations [Imaging cardiac hydatid cysts: 8 cases]. La Presse Médicale, 2006, 35(7-8):1162-1166.

10. Alper H, Yünten R, Sener NR. Intramural hydatid cyst of pulmonary arteries: CT and MR findings. European Journal of Radiology, 1995, 5:666-668.
11. Ben-Hamda K et al. Eighteen-year experience with echinococe cosus of the heart: clinical and echocardiographic features in 14 patients. International Journal of Cardiology, 2003, 91:145-151.

12. McManus DP et al. Echinococcosis. Lancet, 2003, 362:1295-1304.

13. Polat $\mathrm{P}$ et al. Hydatid disease from head to toe. Radiographics, 2003, 23:475-494; quiz 536-537.

14. Gormus $\mathrm{N}$ et al. The clinical and surgical features of right-sided intracardiac masses due to echinococcosis. Heart Vessels, 2004, 19:121-124. 